\title{
On the soft emission mechanism in X-ray pulsars
}

\author{
Paolo Cea' ${ }^{1}$ \\ Received: 9 January 2019 / Accepted: 8 February 2019 / Published online: 18 February 2019 \\ (c) The Author(s) 2019
}

\begin{abstract}
We propose a mechanism responsible for the soft emission in X-ray pulsars. We discuss the origin of the soft emission for isolated X-ray pulsars as well as for soft gamma-ray repeaters and anomalous X-ray pulsars. We also offer an explanation for the spectral features detected in the isolated X-ray pulsar 1E 1207.4-5209.
\end{abstract}

Keywords Pulsars: general $\cdot$ Radiation mechanisms: general $\cdot$ Radiation mechanisms: non-thermal

\section{Introduction}

Pulsars [1] are solar-mass relativistic neutron stars endowed with strong magnetic fields (see e.g. Refs. [2, 3]). Almost all the known pulsars can be interpreted as rapidly spinning, strongly magnetised neutron stars radiating at the expense of their rotational energy (see Ref. [4] for a good account). One notable exception is soft gamma-ray repeaters (SGR) and anomalous X-ray pulsars (AXP) which have been suggested to be magnetars [5-8], namely pulsars with super-strong magnetic fields powered by their own magnetic energy.

Interestingly enough, a number of anomalous X-ray pulsars have recently been detected in the optical-infrared wavelengths (see, for instance, Ref. [9]). Remarkably, there is compelling evidence for an excess of the flux in the infrared band in anomalous X-ray pulsars with respect to the thermal component extrapolated from X-ray data. From the magnetar theory, it is not entirely clear whether one would expect the X-ray and infrared fluxes to be correlated. Such a relationship has been seen for the AXP 1E 2259+586, which has been shown to have correlated X-ray and infrared fluxes, following an X-ray bursting episode [10]. In any case, irrespective of the actual mechanism responsible for the soft emission, this correlation strongly suggests that the optical-infrared emission originates in the magnetosphere. Looking at the broadband energy spectrum of anomalous $\mathrm{X}$-ray pulsars, one realises quickly that the spectra are

Paolo Cea

paolo.cea@ba.infn.it

1 INFN - Sezione di Bari, Via Amendola 173, 70126 Bari, Italy amazingly similar to the ones of isolated X-ray pulsars, like RXJ 1856.5-3754 or RXJ 0720.4-3125.

RXJ 1856.5-3754 and RXJ 0720.4-3125 are the nearest sources among thermally emitting isolated pulsars, and they belong to the group of seven radio-quiet isolated pulsars, nicknamed "The Magnificent Seven", discovered in the ROSAT all-sky survey data (see Ref. [11] for a review). In general, it is remarkable that isolated X-ray pulsars do display a faint soft emission, in excess over the extrapolated $\mathrm{X}$-ray thermal emission, which is best fitted by a non-thermal power law. The origin of this faint emission is puzzling. Nevertheless, our previous considerations point towards a general mechanism in the magnetosphere that is responsible for the faint emission. In other words, it must exist some natural mechanism capable of generating the soft tail of the spectrum of isolated pulsar, anomalous X-ray pulsars and soft gamma-ray repeaters. The main purpose of this paper is to discuss a mechanism which is able to explain the faint emission for isolated pulsars as well as anomalous X-ray pulsars and soft gamma-ray repeaters.

The plan of the paper is as follows. In Sect. 2, by means of quantum mechanical calculations in the quasi-classical limit, we show that strong enough dipolar magnetic fields lead to electrons trapped near the polar cap regions. We also discuss the spectral features in isolated thermal X-ray pulsars. In Sect. 3, we suggest that the electrons trapped in the polar cap regions give rise to a faint non-thermal power-law emission by reprocessing the thermal X-ray photons. Finally, we draw our conclusions in Sect. 4. 


\section{Origin of the soft emission in X-ray pulsars}

Our aim is to illustrate a mechanism able to account for the faint emission that seems ubiquitous in isolated pulsars as well as in anomalous X-ray pulsars and soft gamma-ray repeaters. In our proposal, the power-law emission in the infrared-optical band is due to thermal radiation reprocessed in the magnetosphere by electrons trapped near the magnetic polar caps.

To start with, let us consider the motion of a charged particle in the pulsar magnetic dipolar field. We are assuming the presence of neutral plasma formed by electrons and protons with number densities $n_{\mathrm{e}}=n_{p}$. Now, as is well known, for strong enough magnetic fields the plasma near the stellar surface will be channelled towards the magnetic pole so that we are led to consider the motion of charged particles which are drifting towards the magnetic polar caps. Further, we may consider a polar region with area small enough such that the dipolar magnetic field depends only on the distance from the surface. If the magnetic polar axis is in direction $z$, then charged particles moving towards the stellar surface will feel an almost uniform magnetic field having z-component $B(z)$. Thus, we are led to consider the motion of charge particle in the magnetic field:

$B(z)=-B_{S} \frac{R^{3}}{z^{3}}, \quad z \geq R$,

where, for the sake of definitiveness, we are considering the north magnetic pole where the magnetic field is entering into the stellar surface. Let us consider, firstly, electrons with charge $-e$ and mass $m_{e}$. The electron wave function $\psi(x, y, z)$ can be obtained by solving the Schrödinger equation in the presence of the magnetic field $B(z)$. We are interested in the physical problem where an electron, starting from a distance $z_{0}$ from the star, is approaching the stellar surface. Usually, it is assumed that the magnetic field is almost constant, so that our problem reduces to the well-known motion in an uniform magnetic field. In this case, one gets (we set $\hbar=1$, $\left.c=1, k_{B}=1\right)$ :

$\psi(x, y, z)=\exp \left(-i p_{z} z\right) \phi_{n}(x, y)$,

with energy eigenvalues:

$\varepsilon_{n, p_{z}}=\frac{p_{z}^{2}}{2 m_{e}}+\frac{e B_{S}}{m_{e}}\left(n+\frac{1}{2} \pm \frac{1}{2}\right) \quad n=0,1, \ldots$

However, if $z_{0} \gg R$ the uniform field approximation is no longer valid, but the weakly varying field is more appropriate. In this case, we may write:

$\psi(x, y, z)=\zeta(z) \phi_{n}(x, y)$,

where $\phi_{n}(x, y)$ is the solution of the Schrödinger equation in the weakly varying $B(z)$ :

$$
\begin{aligned}
& \frac{1}{2 m_{e}}\left[-\frac{\partial^{2}}{\partial x^{2}}+e^{2} B^{2}(z) y^{2}+2 i B(z) \frac{\partial}{\partial x}-\frac{\partial^{2}}{\partial y^{2}}\right] \phi_{n}(x, y) \\
& \quad=\varepsilon_{n}(z) \phi_{n}(x, y), \\
& \varepsilon_{n}(z)=\frac{e B(z)}{m_{e}}\left(n+\frac{1}{2} \pm \frac{1}{2}\right) \quad n=0,1, \ldots
\end{aligned}
$$

so that writing the total energy of electrons drifting from $z_{0}$ towards the star as:

$E=\varepsilon_{n}\left(z_{0}\right)+\varepsilon_{\text {drift }}$,

the wave function $\zeta(z)$ satisfies the effective Schrödinger equation:

$\frac{1}{2 m_{e}}\left[-\frac{\mathrm{d}^{2}}{\mathrm{~d} z^{2}}+V_{B}(z)\right] \zeta(z)=\varepsilon_{\mathrm{drift}} \zeta(z)$,

where

$V_{B}(z)=\frac{e B_{S}}{m_{e}} \frac{R^{3}}{z_{0}^{3}}\left(n+\frac{1}{2} \pm \frac{1}{2}\right)\left[\left(\frac{z_{0}}{z}\right)^{3}-1\right] \quad R \leq z \leq z_{0}$.

Inspection of Eq. (9) shows that, as long as the total momentum is not exactly parallel to the magnetic field, electrons will feel a repulsive barrier which at the stellar surface is:

$V_{0}=\frac{e B_{S}}{m_{e}} \simeq 11.6 \mathrm{KeV} B_{12}, \quad B_{12}=\frac{B_{S}}{10^{12} \text { Gauss }}$,

at least. This effect is the quantum mechanical counterpart of the well-known fact that classical charges moving towards regions with increasing magnetic field strength are subject to a repulsive force. On the other hand, when we consider protons, the minimal height of the magnetic barrier is:

$V_{0}=\frac{e B_{S}}{m_{p}} \simeq 6.3 B_{12} \mathrm{eV}$.

In other words, protons drifting towards the surface almost do not feel any barrier. As a consequence, there is an accumulation of positive charge on the surface. Note that on the surface of the star there is a positively charged layer which is able to support a thin crust of ordinary matter. Thus, protons do not dissolve into the star, but they are trapped in the atmosphere of electrons which extends over a distance $\delta \sim 10^{3}$ fermis beyond the edge. Let $n$ be the number density of trapped protons, then on the surface of the star there is a surface charge density $\sigma \simeq e \delta n$ which, in turn, gives rise to an uniform electric field. It follows that electrons that are moving towards the star are subject to both the repulsive 
magnetic potential and the attractive electric potential so that the effective potential is:

$$
V(z)=\frac{e B_{S}}{m_{e}} \frac{R^{3}}{z_{0}^{3}}\left(n+\frac{1}{2} \pm \frac{1}{2}\right)\left[\left(\frac{z_{0}}{z}\right)^{3}-1\right]+4 \pi e^{2} n \delta z, \quad R \leq z \leq z_{0} .
$$

In the case of minimal magnetic barrier, using Eq. (10) we rewrite Eq. (12) as:

$$
\begin{aligned}
& V(z) \simeq 11.6 \mathrm{KeV}_{12} \frac{R^{3}}{z_{0}^{3}}\left[\left(\frac{z_{0}}{z}\right)^{3}-1\right] \\
&+0.23 \mathrm{KeV} n_{13} \frac{z}{R}, \quad 1 \leq \frac{z}{R} \leq \frac{z_{0}}{R},
\end{aligned}
$$

where $n_{13}=\frac{n}{10^{13} \mathrm{~cm}^{-3}}$. In Fig. 1, we display the effective potential Eq. (13) assuming $z_{0} \simeq 10 R$. We see that the effective potential $V(z)$ displays a minimum at $z=\bar{z}$ where the repulsive magnetic force is balanced by the attractive electric force. Then, electron is trapped above the polar cap at a distance of order $\bar{z}$. To determine the energy spectrum, we need to solve the Schrödinger equation with the effective potential:

$\frac{1}{2 m_{e}}\left[-\frac{\mathrm{d}^{2}}{\mathrm{~d} z^{2}}+V(z)\right] \zeta(z)=\varepsilon_{\mathrm{drift}} \zeta(z)$.

We may adopt the harmonic approximation to the potential by expanding around $\bar{z}$. A straightforward calculation gives:

$\bar{z} \simeq 3.5 R B_{12}^{\frac{1}{4}} n_{13}^{-\frac{1}{4}}, \quad \bar{V} \equiv V(\bar{z}) \simeq 1.08 \mathrm{KeV} B_{12}^{\frac{1}{4}} n_{13}^{\frac{3}{4}}$.

Moreover:

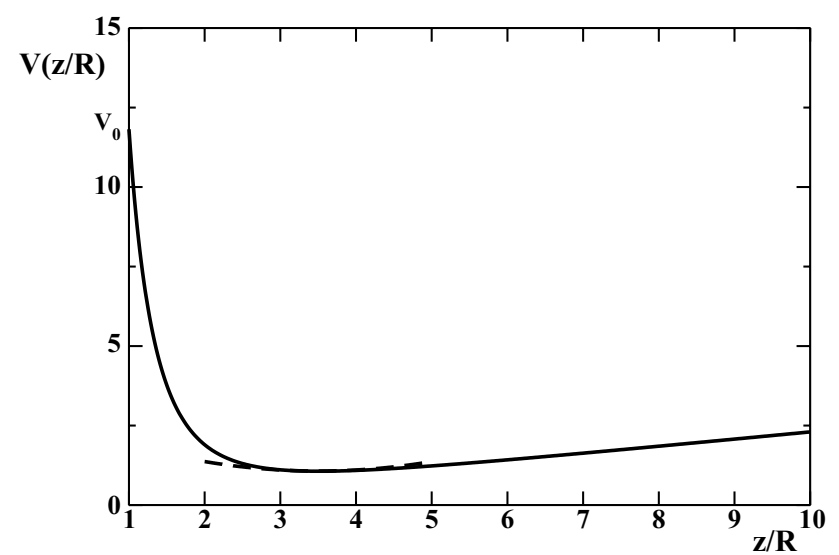

Fig. 1 The full line is the effective potential Eq. (13) felt by the electrons drifting towards the stellar surface in the polar cap regions assuming $z_{0}=10 R, n_{13}=1, B_{12}=1$. The dashed line is the harmonic approximation to the potential
$R^{2} V^{\prime \prime}(\bar{z}) \simeq 0.261 \mathrm{KeV} B_{12}^{-\frac{1}{4}} n_{13}^{\frac{5}{4}}$.

Note that, as long as $z_{0} \gg R, \bar{z}, \bar{V}$ and $V^{\prime \prime}(\bar{z})$ do not depend on $z_{0}$. In this approximation, $\zeta(z)$ satisfies the harmonic oscillator equation centred at $\bar{z}$ with frequency:

$\omega_{m} \simeq 0.46 \times 10^{-12} \mathrm{eV} B_{12}^{-\frac{1}{8}} n_{13}^{\frac{5}{8}}$.

Thus, we find for the drift energy the quasi-continuum spectrum:

$\varepsilon_{\mathrm{drift}, j}=\bar{V}+\omega_{m}\left(j+\frac{1}{2}\right), \quad j \geq 0$.

Let us pause to briefly summarise our results. Our quantum mechanical treatment of charges which are drifting towards the star in the weakly varying dipolar magnetic field has shown that electrons feel a huge magnetic barrier. On the other hand, the magnetic barrier is reduced by a factor $\frac{m_{e}}{m_{p}}$ for protons. As a consequence, protons are free to reach the stellar surface, where they are trapped in the electron atmosphere, while electrons are repelled into the magnetosphere. The resulting charge separation produces an electric field which traps electrons at a distance $\sim \bar{z}$. Obviously, the neutrality of the plasma implies that the number densities of trapped electrons and protons are equal. Thus, we are led to the following picture. We have electrons which oscillate around $\bar{z}$ along the magnetic field. On the other hand, these electrons feel the magnetic field $B(\bar{z})$, which now is truly almost constant so that, if $\omega_{B}$ is the cyclotron frequency at $\bar{z}$, then using Eq. (15) we get:

$\omega_{B}=\frac{e B(\bar{z})}{m_{e}} \simeq 0.268 \mathrm{KeV} B_{12}^{\frac{1}{4}} n_{13}^{\frac{3}{4}}$.

Therefore, the energy spectrum of these electrons is given by:

$E_{n, j}=\bar{V}+\omega_{B} n+\omega_{m}\left(j+\frac{1}{2}\right), \quad n, j \geq 0$.

In Fig. 2, we illustrate schematically the spectrum Eq. (20). According to Eq. (20), the spectrum comprises discrete Landau levels with cyclotron frequency $\omega_{B}$ and an almost continuum associated with the drifting motion. As discussed in the next section, electrons perform radiative transition between Landau levels. These transitions are responsible for absorption and spectral features observed in isolated X-ray pulsars, anomalous X-ray pulsars and soft gamma-ray repeaters. Moreover, we see that there are also transitions where electrons absorb thermal photons with frequency $\omega \sim \omega_{B}$ and emit photons with frequencies $\omega^{\prime} \ll \omega$. These radiative transitions give rise to the 


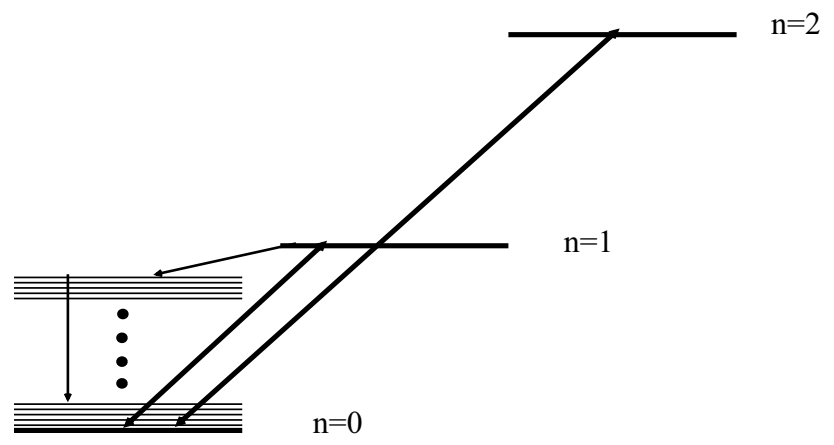

Fig. 2 Energy spectrum for electrons trapped in the magnetosphere near the polar caps. Heavy lines are the Landau levels with cyclotron frequency $\omega_{B}$. Light lines are the quasi-continuum spectrum Eq. (18). Heavy and light arrows indicate the allowed radiative transitions

soft spectrum. Before addressing the problem of the soft spectrum, let us discuss the puzzling absorption features detected in the isolated X-ray pulsar 1E 1207.4-5209 [12]. The spectrum of 1E 1207.4-5209 shows three distinct features, regularly spaced at $0.7 \mathrm{KeV}, 1.4 \mathrm{KeV}$ and $2.1 \mathrm{KeV}$, plus possibly a fourth at $2.8 \mathrm{KeV}$. These features vary in phase with the star rotation. Indeed, it turns out that the X-ray source pulsation is largely due to the phase variation of the lines with the pulsar rotation. The most natural and logical explanation for the observed features is cyclotron resonant absorption. In this case, the fundamental cyclotron frequency is $0.7 \mathrm{KeV}$. Thus, the inferred magnetic field is $\sim 8 \times 10^{10}$ Gauss for electrons and $\sim 2 \times 10^{14}$ Gauss for protons. However, the magnetic field inferred from the spin parameters turns out to $B_{\mathrm{S}} \simeq 2.4 \times 10^{12}$ Gauss. Obviously, as noted in Ref. [12] electron cyclotron scattering at a distance $3-4 R$ above the pulsar surface would fit all the observations. Remarkably, using the inferred magnetic field $B_{S}$ and Eq. (19), we get:

$\omega_{B} \simeq 0.7 \mathrm{KeV}, \quad B_{S, 12} \simeq 2.4 \quad n_{13} \simeq 2.69$.

Moreover from Eq. (15), we get $\bar{z} \simeq 3.4 R$, in accord with observations. Finally, we stress that the presence of the almost continuum spectrum associated with the drifting motion along the magnetic field leads naturally to rather broader spectral features in agreement with several observations. Even though it is beyond the aim of the present section an accurate comparison with available data, we would like to stress that our proposal seems to be in satisfying qualitative agreement with observations. Indeed, absorption features have been found in the thermal emission of several isolated X-ray pulsars which range in $0.2-0.7 \mathrm{KeV}$. The thermal spectrum of isolated $\mathrm{X}$-ray pulsar is a blackbody with typical temperature $T \sim 0.1 \mathrm{KeV}$. Now Eq. (19) shows that for typical pulsar magnetic field $B_{12} \sim 1$ the electrons trapped at $\sim \bar{z}$ are able to absorb thermal photons in the observed range. Note that for magnetars the typical blackbody temperature is $T \sim 1.0 \mathrm{KeV}$ (the relevant temperature is the polar cap blackbody temperature) and $B_{12} \sim 10^{2}$ so that Eq. (19) gives $\omega_{B} \sim 0.85 \mathrm{KeV}$, indicating that also for magnetars the trapped electrons may efficiently absorb the thermal $\mathrm{X}$-ray photons from the polar caps.

\section{The soft spectrum}

Let us discuss, now, the radiative transitions from trapped electrons. To evaluate the transition rates, we need to evaluate (for instance, see Refs. [13, 14]):

$\left|\frac{i e}{m_{e}}\left\langle n^{\prime}, j^{\prime}|\exp (-i \mathbf{k} \cdot \mathbf{r}) \mathbf{l} \cdot \nabla| n, j\right\rangle\right|^{2}$

where

$|n, j\rangle=\zeta_{j}(z) \phi_{n}(x, y), \quad \mathbf{l} \cdot \mathbf{k}=0$,

$\mathbf{k}$ and $\mathbf{I}$ being the photon momentum and polarisation, respectively. Without loss in generality, we may write:

$\mathbf{k}=\left(k_{\perp}, 0, k_{3}\right), \quad \mathbf{l}=(0,1,0)$,

so that

$\left|\frac{i e}{m_{e}}\left\langle n^{\prime}, j^{\prime}|\exp (-i k \cdot r) l \cdot \nabla| n, j\right\rangle\right|^{2}=\frac{e^{2}}{m_{e}^{2}} I_{n^{\prime}, n} J_{j^{\prime}, j}$,

where

$I_{n^{\prime}, n}=\left|\left\langle n^{\prime}\left|\exp \left(-i k_{\perp} x\right) \frac{\partial}{\partial y}\right| n\right\rangle\right|^{2}$,

$J_{j^{\prime}, j}=\left|\left\langle j^{\prime}\left|\exp \left(-i k_{3} z\right)\right| j\right\rangle\right|^{2}$.

Obviously, we have also:

$|\mathbf{k}|=E_{n^{\prime} j^{\prime}}-E_{n, j}=\omega_{B}\left(n^{\prime}-n\right)+\omega_{m}\left(j^{\prime}-j\right)$.

We are interested in processes where trapped electrons absorb thermal photons with energy $\sim \omega_{B}$ (heavy arrows in Fig. 2) and emit photons with $|\mathbf{k}| \ll \omega_{B}$ (light arrows in Fig. 2). Since $\omega_{m} \sim 10^{-12} \mathrm{eV}$, from Eq. (28) it follows that $\Delta j \gg 1$. Then, the transitions $j \rightarrow j^{\prime}$ cannot be induced by the electromagnetic field. Indeed, these transitions are induced by thermal collisions. To see this, we note that for $j \gg 1$ the wave function $\zeta(z)$ is quasi-classical:

$\zeta(z) \sim \exp [-i p(z) z]$

where $p(z)$ is the quasi-classical momentum. Now, Eq. (27) means that $J_{j^{\prime}, j}$ is different from zero if the 
momentum is conserved. However, for vastly different quasi-momenta the overlap of the quasi-classical wave function is very small so that we are lead to the conclusion that $\left|k_{3}\right| \ll\left|k_{\perp}\right|$. As a consequence, we see that the transitions must be induced by collisions, so that to a good approximation we may set $\left|J_{j^{\prime}, j}\right| \simeq 1$. To evaluate $I_{n^{\prime}, n}$, we need the wave functions $\phi_{n}(x, y)$ :

$\phi_{n}(x, y)=\frac{\exp \left(-i p_{x} x\right)}{\sqrt{2 \pi}} \sqrt{\frac{m_{e} \omega_{B}}{n ! 2^{n}}} \frac{1}{\pi^{\frac{1}{4}}}$
$\exp \left[-\frac{m_{e} \omega_{B}}{2}\left(y-\frac{p_{x}}{m_{e} \omega_{B}}\right)^{2}\right] H_{n}\left[\sqrt{m_{e} \omega_{B}}\left(y-\frac{p_{x}}{m_{e} \omega_{B}}\right)\right]$.

The most important transition is for $n^{\prime}=1, n=0$. A standard calculations gives:

$I_{1,0}=\frac{m_{e} \omega_{B}}{2}\left(1-\frac{k_{\perp}^{2}}{2 m_{e} \omega_{B}}\right)^{2} \exp \left[-\frac{k_{\perp}^{2}}{2 m_{e} \omega_{B}}\right]$,

so that

$$
\begin{aligned}
& \left|\frac{i e}{m_{e}}\left\langle n^{\prime}, j^{\prime}|\exp (-i \mathbf{k} \cdot \mathbf{r}) \mathbf{l} \cdot \nabla| n, j\right\rangle\right|^{2} \\
& \quad \simeq \frac{e^{2} \omega_{B}}{2 m_{e}}\left(1-\frac{k_{\perp}^{2}}{2 m_{e} \omega_{B}}\right)^{2} \exp \left[-\frac{k_{\perp}^{2}}{2 m_{e} \omega_{B}}\right] .
\end{aligned}
$$

Note that in our approximation $|\mathbf{k}|=k \simeq\left|k_{\perp}\right| \ll \omega_{B}$. Thus, we have:

$\left|\mathcal{A}_{i f}\right|^{2} \equiv\left|\frac{i e}{m_{e}}\left\langle n^{\prime}, j^{\prime}|\exp (-i \mathbf{k} \cdot \mathbf{r}) \mathbf{l} \cdot \boldsymbol{\nabla}| n, j\right\rangle\right|^{2} \simeq \frac{e^{2} \omega_{B}}{2 m_{e}}$.

It is clear that Eq. (33) leads to a power-law emission flux with the cut-off $k \ll \omega_{B}$. Moreover, to get the flux we need to assume some energy distribution for the trapped electrons, which, however, goes beyond the aim of the present paper. Nevertheless, there is a general aspect which is worthwhile to stress. We have seen that the soft spectrum in isolated pulsar can be understood as thermal photons reprocessed by electrons trapped in the magnetosphere above the polar caps. These electrons absorb thermal photons with frequency $\sim \omega_{B}$ and emit photons with frequencies $\ll \omega_{B}$ so that the number of these electrons is proportional to the number of thermal photons with energy $\sim \omega_{B}$. We know that $\omega_{B} \sim T$, where $T$ is the blackbody temperature. As a consequence, recalling that the number density of thermal photons scales as $\sim T^{3}$, we have that the soft fluxes in magnetars should be about a factor $\left(T_{\text {magnetar }} / T_{\text {pulsar }}\right)^{3} \sim 10^{3}$ greater than the one in isolated $\mathrm{X}$-ray pulsars, in fair agreement with observations [15].
Let us conclude by roughly estimating the soft flux $F_{\omega}$. The probability for emission of a photon in the frequency range $\omega$, $\omega+\mathrm{d} \omega$ is:

$\frac{\mathrm{d} P}{\mathrm{~d} t \mathrm{~d} \omega}=\frac{\omega}{2 \pi}\left|\mathcal{A}_{i f}\right|^{2} \delta\left[\omega-\left(E_{1, j^{\prime}}-E_{0, j}\right)\right]$,

where we used $\omega=|\mathbf{k}|=k \simeq\left|k_{\perp}\right|$, and the delta function ensures the energy conservation Eq. (28). Summing over the degenerate final states of the almost continuum spectrum, and using $\mathrm{d} \omega=\omega_{m} \mathrm{~d} j^{\prime}$, we get:

$\frac{\mathrm{d} P}{\mathrm{~d} t \mathrm{~d} \omega} \simeq \frac{1}{2 \pi} \frac{\omega}{\omega_{m}} \frac{e^{2} \omega_{B}}{2 m_{e}}$.

Finally, the spectral flux is obtained multiplying by the photon energy $\omega$ and by the number of active electrons. Let $n_{\text {act }}$ be the number density of active electrons, we have:

$F_{\omega} \simeq \frac{1}{2 \pi} \frac{\omega^{2}}{\omega_{m}} \frac{e^{2} \omega_{B}}{2 m_{e}} n_{\mathrm{act}} V, \quad \omega \ll \omega_{B}$,

where $V$ is the volume of the emitting region. Note that $F_{\omega}$ has a Rayleigh-Jeans power law form with an upper cut-off which, however, cannot be easily estimated without a precise knowledge of electron energy distribution. Using Eqs. (17) and (19), we rewrite Eq. (36) as:

$F_{\omega} \simeq 8.2 \times 10^{-6} \frac{\mathrm{ergs}}{\operatorname{sec~Hz}}\left(\frac{\omega}{1 \mathrm{eV}}\right)^{2} B_{12}^{\frac{3}{8}} n_{13}^{\frac{1}{8}} n_{\text {act }} V$.

The number density of active electrons is not easily estimated without the knowledge of the energy distribution of electrons trapped into the magnetosphere. In general, $n_{\text {act }}$ depends on the number of thermal photons with energy $\omega \sim \omega_{B}$. Nevertheless, we may estimate the needed number density $n_{\text {act }}$ by comparing with observed soft fluxes. For typical isolated X-ray pulsar with $B_{12} \sim 1$ and $n_{13} \sim 1$, we infer:

$F_{\omega} \simeq 10^{13} \frac{\mathrm{ergs}}{\mathrm{sec} \mathrm{Hz}}, \quad \omega \simeq 1 \mathrm{eV}$.

Hence, assuming for the emitting volume the reasonable value $V \sim 10^{10} \mathrm{~cm}^{3}$, from Eq. (37) we get:

$n_{\text {act }} \sim 10^{8} \mathrm{~cm}^{-3} \sim 10^{-5} n$.

It is interesting to compare Eq. (39) with the number density of active electrons obtained assuming that trapped electrons have an uniform distribution. In this case, observing that the probability for transitions from the $n=0$ to $n=1$ Landau levels is given by Eq. (32) with $k_{\perp} \simeq \omega_{B}$, we get:

$n_{\mathrm{act}} \simeq \frac{e^{2} \omega_{B}}{2 m_{e}} n$

so that for typical isolated X-ray pulsars we have: 
$n_{\text {act }} \simeq 2.4 \times 10^{-5} n$,

which, indeed, is in reasonable agreement with our estimate Eq. (39).

\section{Conclusions}

In the present note, we have developed an effective mechanism for the soft emission in isolated X-ray pulsars endowed with strong enough dipolar magnetic field. We solved the Schrödinger equation in the quasi-classical approximation for electrons drifting towards the polar caps due to a strong enough dipolar magnetic field. We found that the drifting electrons can be trapped at distances of a few stellar radii. We also pointed out that the trapped electrons could absorb thermal X-ray photons and, therefore, could give rise to a non-thermal power-like faint emission in the optical-infrared band. Even though we did not attempt a precise quantitative comparison with observational data, we found that our proposal compares reasonably well with observations. It should be stressed, however, that we employed several oversimplifications to better illustrate our proposal. Firstly, the structure of the magnetosphere around a pulsar is exceedingly complicated. In fact, only recently it has been displayed consistent numerical solutions ( see Refs. [16, 17] and references therein). As a consequence to obtain reliable results to be compared with observations, we need to address the problem by means of magnetohydrodynamic numerical simulations. In addition, our quantum-mechanical treatment is questionable in pulsars with surface magnetic fields exceeding the quantum critical field $B_{\mathrm{Q}} \simeq 4.414 \times 10^{13} \mathrm{G}$ at which the cyclotron energy equals the electron rest mass. In these cases, it should be employed a fully relativistic treatment (see Ref. [18] for an exhaustive review). We hope to report progress on these subjects in a future paper.

Open Access This article is distributed under the terms of the Creative Commons Attribution 4.0 International License (http://creativeco mmons.org/licenses/by/4.0/), which permits unrestricted use, distribution, and reproduction in any medium, provided you give appropriate credit to the original author(s) and the source, provide a link to the Creative Commons license, and indicate if changes were made.

\section{References}

1. Hewish, A., et al.: Observation of a rapidly pulsating radio source. Nature 217, 709 (1968)

2. Manchester, R.N., Taylor, J.H.: Pulsars. W. H. Freeman and Company, London (1977)

3. Shapiro, S.L., Teukolsky, S.A.: Black Holes, White Dwarfs, and Neutron Stars. Wiley, Hoboken (1983)

4. Becker, W.: X-Ray emission from pulsars and neutron stars. In: Becker, W. (ed.) Neutron Stars and Pulsars (Astrophysics and Space Science Library), vol. 357. Springer, Berlin (2009)

5. Duncan, R.C., Thompson, C.: Formation of very strongly magnetized neutron stars-Implications for gamma-ray bursts. Astrophys. J. 392, L9 (1992)

6. Duncan, R.C., Thompson, C.: Neutron star dynamos and the origins of pulsar magnetism. Astrophys. J. 408, 194 (1993)

7. Duncan, R.C., Thompson, C.: The soft gamma repeaters as very strongly magnetized neutron stars-I: radiative mechanism for outbursts. Monthly Not. R. Astron. Soc. 275, 255 (1995)

8. Kaspi, V.M., Beloborodov, A.M.: Magnetars. Ann. Rev. Astr. Astrophys. 55, 261 (2017)

9. Israel, G. et al.: Young neutron stars and their environments. In: Camilo, F., Gaensler, B.M. (eds.) IAU Symposium, vol. 218. Astronomical Society of the Pacific, San Francisco (2004)

10. Tam, C., Kaspi, V., van Kerkwijk, M., Durant, M.: Correlated infrared and X-ray flux changes following the 2002 June outburst of the anomalous X-ray pulsar 1E 2259+586. Astrophys. J. 617, L53 (2004)

11. Haberl, F.: The magnificent seven: magnetic fields and surface temperature distributions. Astrophys. Space Sci. 308, 181 (2007)

12. Bignami, G.F., Caraveo, P.A., De Luca, A., Mereghetti, S.: The magnetic field of an isolated neutron star from X-ray cyclotron absorption lines. Nature 423, 725 (2003)

13. Wallace, W.H.: Radiation Processes in Astrophysics. MIT Press, Cambridge (1977)

14. Rybicki, G.B., Lightman, A.P.: Radiative Processes in Astrophysics. Wiley, Hoboken (1979)

15. Coti Zelati, F., Rea, N., Pons, J.A., Campana, S., Esposito, P.: Systematic study of magnetar outbursts. Monthly Not. R. Astron. Soc. 474, 961 (2018)

16. Arons, J.: Pulsar emission: where to go. In: Becker, F.W. (ed.) Neutron Stars and Pulsars. Astrophysics and Space Science Library, vol. 357. Springer, Berlin (2009)

17. Cerutti, B., Beloborodov, A.M.: Electrodynamics of pulsar magnetospheres. Space Sci. Rev. 207, 111 (2017)

18. Harding, A.K., Lai, D.: Physics of strongly magnetized neutron stars. Rep. Prog. Phys. 69, 2631 (2006)

Publisher's Note Springer Nature remains neutral with regard to jurisdictional claims in published maps and institutional affiliations. 